\title{
Spatio-temporal Analysis of Electroconvection in Nematics
}

\author{
Heidrun Amm, Maren Grigutsch, and Ralf Stannarius \\ Universität Leipzig, Fak. Physik und Geowiss., Linnéstr. 5, D-04103 Leipzig
}

Z. Naturforsch. 53 a, 117-126 (1998); received December 10, 1997

\begin{abstract}
We investigate electroconvection in nematic liquid crystals by means of optical microscopy. Time resolved optical images are used to study the director dynamics. For the first time we present instant images in the dielectric regime. A numerical simulation of the optical transmission patterns is performed on the basis of Fermat's principle. In the instant images of dielectric rolls, the periodicity of the observed optical pattern is equal to the wavelength $\lambda_{0}$ of the convection rolls. The well known low contrast 'stationary' optical texture observed in conventional experiments results from time averaging of these instant images; its wavelength is $\lambda_{0} / 2$.
\end{abstract}

Key words: Liquid Crystals; Pattern Formation; Electrohydrodynamic Instability;

Optical Microscopy.

\section{Introduction}

Electrohydrodynamic convection (EHC) has been extensively investigated both experimentally and theoretically since its discovery in 1963 by Williams and Kapustin [1]. For a recent review we refer the reader to [2]. Being easily observable and controllable in the laboratory, EHC serves as a standard system for convection patterns. From its first theoretical explanation by Carr and Helfrich [3] the theoretical analysis has been developed consequently from a one-dimensional [4] to a three-dimensional description [5] and weak electrolyte model (WEM) [6]. Within these models, most features of the observed patterns are nowadays well understood. Small periodic director fluctuations in connection with the anisotropic conductivity of the nematic lead to the formation of an in-plane periodically modulated charge field that in turn augments the director deflection via flow coupling. The dynamic properties of these coupled director and charge fields determine the character of the convection structures to a great extend.

In the low frequency conduction regime, different regular instabilities are found, as for example normal and oblique rolls (respective to the director easy axis) with stationary, fluctuating or travelling dynamics. Their optical textures are well described. Characteristic wavelengths are roughly equal to the cell gap.

Reprint requests to Dr. Ralf Stannarius; Fax: +49 34197 32599, E-mail: stanni@lucifer.exphysik.uni-leipzig.de.
At high voltages, a scattering state (DSM) is reached. A characteristic feature of the conduction regime is the symmetry of director field and charge field with respect to the driving $\mathrm{AC}$ voltage. The charge field alternates its sign with the driving electric field $E$ while the director field may be modulated in time but retains its sign.

A completely different behaviour is found above some characteristic material-dependent frequency $f_{\mathrm{c}}$. This cut-off frequency $f_{\mathrm{c}}$ separates the low frequency conduction regime from the high frequency dielectric regime. In the dielectric regime, the director field reorients with the driving frequency $f_{0}$, while the charge field keeps its sign. One observes normal dielectric rolls (DR) at the onset voltage. Their wavelength is frequency dependent and usually much shorter than that of conductive rolls. The optical contrast is much weaker than that of the conductive patterns.

While theory can completely predict the director field of EHC in time and space both in the conduction and dielectric regime, experiments have mostly been restricted to the observation of stationary optical patterns or other time averages of the involved structures. The oscillatory behaviour of director structures in the dielectric regime was established already in early experiments $[7,8]$. In a study of DR using stroboscopic illumination, Schneider et al. [9] have shown experimentally that the amplitudes of optical contrast are strongly time dependent as a consequence of the dynamics of the director field. They measured the time-periodic contrast oscillations and determined 


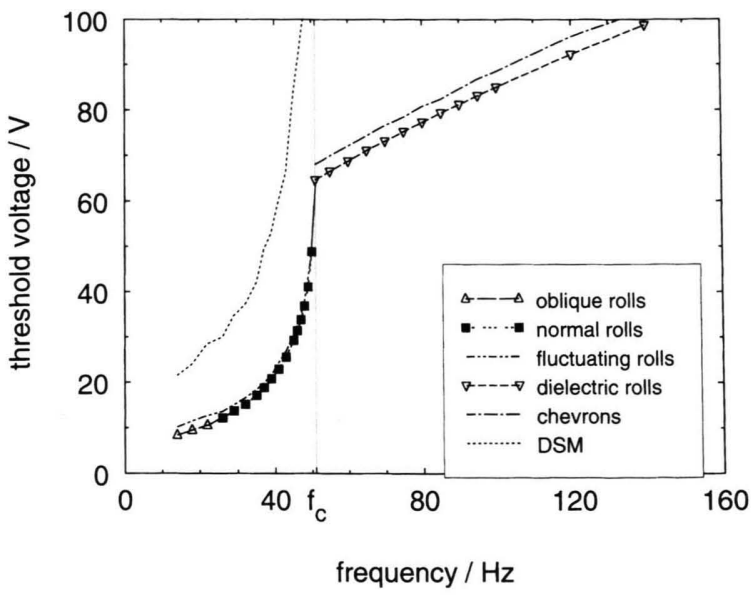

Fig. 1. Threshold curve of the first instability of EHC in Mischung 5 for square wave (SQ) excitation.

their phase shift with respect to the driving electric field.

In our experiments, we study a nematic with very low cut-off frequency by means of a fast line scan camera. This allows us to record directly the time dependent optical images in a cross section of the texture. Such a line scan technique was used, for example, in previous work of Rehberg et al.[10] who studied the dynamics of stationary and travelling rolls. In their experiments, each data point represents a timeaverage over several periods of the driving AC voltage, and the long-term dynamics was investigated. In contrast, we are interested here in the short-term dynamics, that is the texture evolution during each period of the driving field. We sample data during the cycle of the AC driving field and obtain momentary images of the convection patterns. By means of digital image processing, these spatio-temporal optical patterns are analyzed and related to the conventional 'stationary' textures. A simple model for light propagation in a weakly distorted nematic layer is used to simulate the optical properties of dielectric rolls.

\section{Experiment}

The nematic liquid crystal investigated (Mischung 5) is a four component mixture of disubstituted phenyl-benzoates

$$
\begin{aligned}
& n(\mathrm{O})-\mathrm{O} m: \mathrm{C}_{n} \mathrm{H}_{2 n+1}-(\mathrm{O})-\bigcirc-\mathrm{COO}-\bigcirc- \\
& -\mathrm{O}-\mathrm{C}_{m} \mathrm{H}_{2 m+1}
\end{aligned}
$$

1O-06: $22.0 \%, 50-08: 30.3 \%$, 6O-O7: $13.3 \%$, 6-O4: $34.4 \%$.
It provides a large nematic range $\left(70.5^{\circ} \mathrm{C}\right.$ to below RT) and has been extensively characterized (e. g. [11]). Its dielectric anisotropy $\epsilon_{\|}-\epsilon_{\perp}$ is negative. At $30{ }^{\circ} \mathrm{C}$, we have determined $K_{11}=14.9 \mathrm{pN}, K_{22}=$ $6.10 \mathrm{pN}, K_{33}=13.76 \mathrm{pN}, \gamma_{1}=0.36 \mathrm{~Pa}$ s from the static and the dynamic Fréedericksz transition, and $\sigma_{\|}=1.3 \cdot 10^{-8}(\Omega \mathrm{m})^{-1}, \sigma_{\perp}=1.0 \cdot 10^{-8}(\Omega \mathrm{m})^{-1}$, $\epsilon_{\|}=6.24, \epsilon_{\perp}=6.67[12]$ from dielectric spectroscopy.

We use the standard experimental setup, a planar commercial cell (LINKAM) with transparent ITO electrodes and rubbed polyimide coating, $48.5 \mu \mathrm{m}$ cell gap and $5 \mathrm{~mm} \times 5 \mathrm{~mm}$ electrode area. Temperature control of the sample cell is achieved by a LINKAM hot stage. The sample is observed in transmission, using a JENAPOL-d polarizing microscope with normally incident white light polarized along the director easy axis (parallel polarizers along the rubbing direction of the cell).

Stationary video images were taken with a conventional CCD camera. The time resolved images have been recorded with a fast 512 pixel line scan camera (DALSA Cl-C3-0512M) and digitized with an OCULUS OD 500 frame grabber. The highest time resolution of the spatio-temporal images is limited by the CCD exposure time to at least $250 \mu$ s per line. The spatial resolution is $\approx 0.7 \mu \mathrm{m}$ per camera pixel.

\section{Experimental Results}

The sample shows a large variety of EHC patterns $[12,13]$. At low frequencies, the first instability are oblique rolls, the angle between rolls and director easy axis decreases with increasing frequency. At intermediate frequencies, stationary normal rolls become the first instability. Slightly below the cut-off frequency, travelling rolls are observed. With increasing voltages we find fluctuating rolls and finally the transition to DSM. Above the cut-off $f_{\mathrm{c}}$, the first instability are DR which transform into a regular defect texture (chevrons) only a few percent above the onset threshold voltage. The threshold diagram of the first instability of Mischung 5 at $30{ }^{\circ} \mathrm{C}$ is shown in Figure 1 . We have used square wave excitation unless otherwise specified in the text. Due to the temperature dependence of the material parameters, in particular of the viscosities and conductivities, the cut-off is strongly temperature dependent while the general structure of the phase diagram is preserved at all temperatures in the nematic phase. 
a)

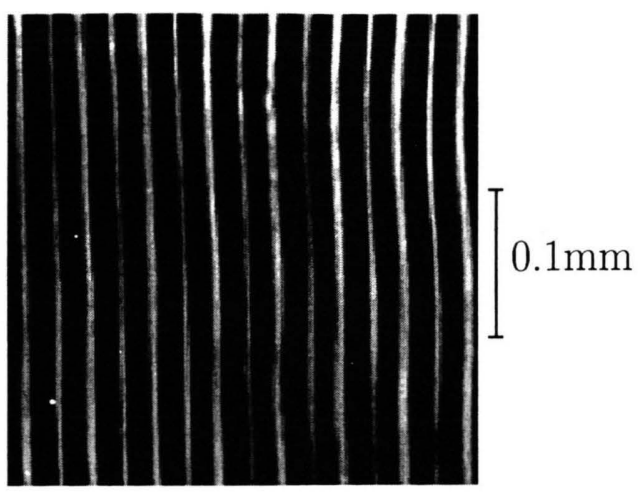

b)
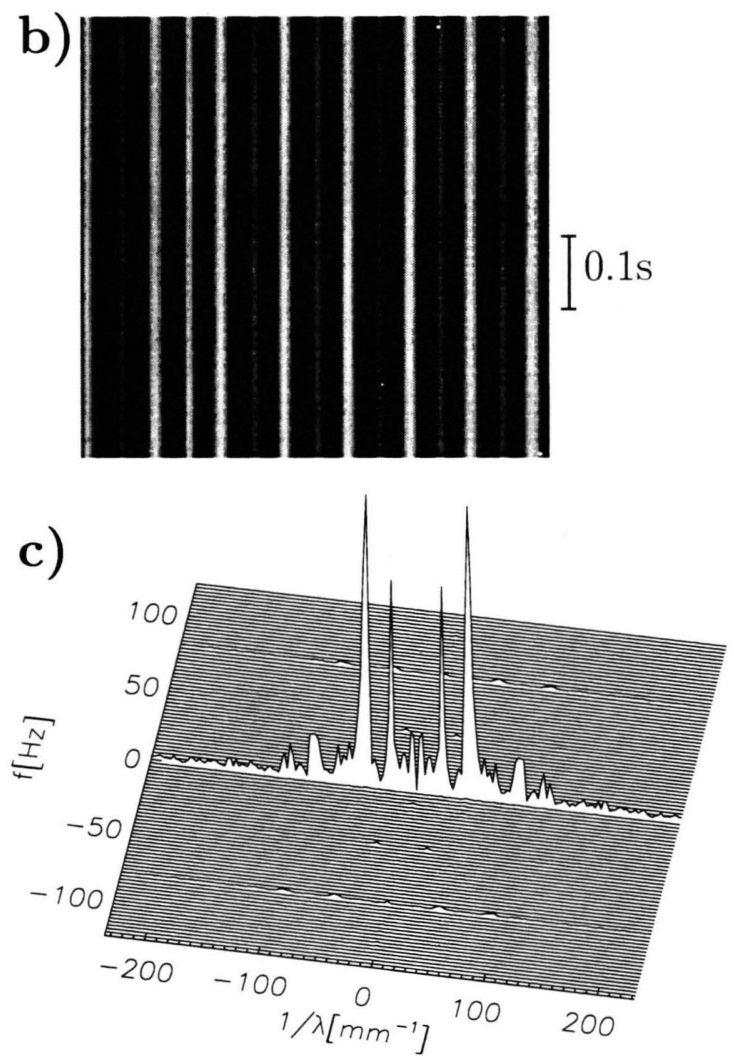

Fig. 2. Normal conductive rolls, $T=30{ }^{\circ} \mathrm{C}, f=40 \mathrm{~Hz}$ SQ, $U=27.5 \mathrm{~V}$, a) stationary optical image $(x, y)$, b) spatiotemporal image $(x, t)$, cut along the director easy axis, time runs from bottom to top, c) 2D Fourier transform of the $(x, t)$ image.

In the following, we show the qualitative differences of the experimental images taken in four regions of the phase diagram. In our coordinate system, the $z$ axis is normal to the cell, $x$ and $y$ are in the cell plane and the $x$-axis (orientation of the line scan camera) is chosen along the director easy axis, i.e. perpendicular to normal rolls. Figure 2 shows typical images of conductive normal rolls near the onset. Together with the transmission pattern $I(x, y)$ in Fig. 2 a and the spatiotemporal image $I(x, t)$ in Fig. $2 \mathrm{~b}$, we present a digital 2D Fourier transform (power spectrum)

$$
\mathcal{F}(k, f)=\left|\int e^{-2 \pi i f t} e^{-2 \pi i k x} I(x, t) \mathrm{d} x \mathrm{~d} t\right|
$$

with $k=1 / \lambda$, in Figure 2c. In all Fourier transforms we suppress the integral intensity peak $(\mathcal{F}(0,0))$ and concentrate on the qualitative characterization of the spatio-temporally inhomogeneous patterns. Phase relations between driving field and optical contrast have not been considered here, they were extensively studied by Schneider et al. [9].

The FT of the stationary image is represented in the horizontal $f=0$ axis of Figure 2c. Peaks on the $1 / \lambda$ axis reflect the periodicity of the conductive rolls with wavelength $\lambda_{0}$, with harmonics originating mainly from the nonlinear optical characteristics. The peak intensity provides a measure of the optical contrast (intensity modulation) of the corresponding mode. The transmission texture is basically generated by refraction of light in the modulated director field. As is well known, the image depends strongly on the microscopic focus. Linear, quadratic and higher order modulations of the transmitted intensity by the periodic director pattern are involved, and the relative strengths of the linear and quadratic effects of the director modulation on the light deflection can differ considerably for different focus planes. In our experiments, the microscopic focus was chosen for convenience at the upper edge of the nematic layer. In the conduction regime, the choice of a different focus may change the spatial optical pattern but is not important for the discussion of the temporal characteristics.

A characteristic feature of the FT spectrum is that additional peaks in the spectrum at frequencies $f \neq 0$ are negligibly small. The optical contrast (and consequently the director modulation also) is nearly stationary, as expected from theory. We compare this picture with Figs. $3 \mathrm{a}-\mathrm{c}$ taken at higher voltages, still in the conduction regime. One recognizes additional peaks growing at frequencies $f= \pm 2 f_{0}$, where $f_{0}$ is the excitation frequency. The pattern contrast is increasingly modulated with the second harmonics of 

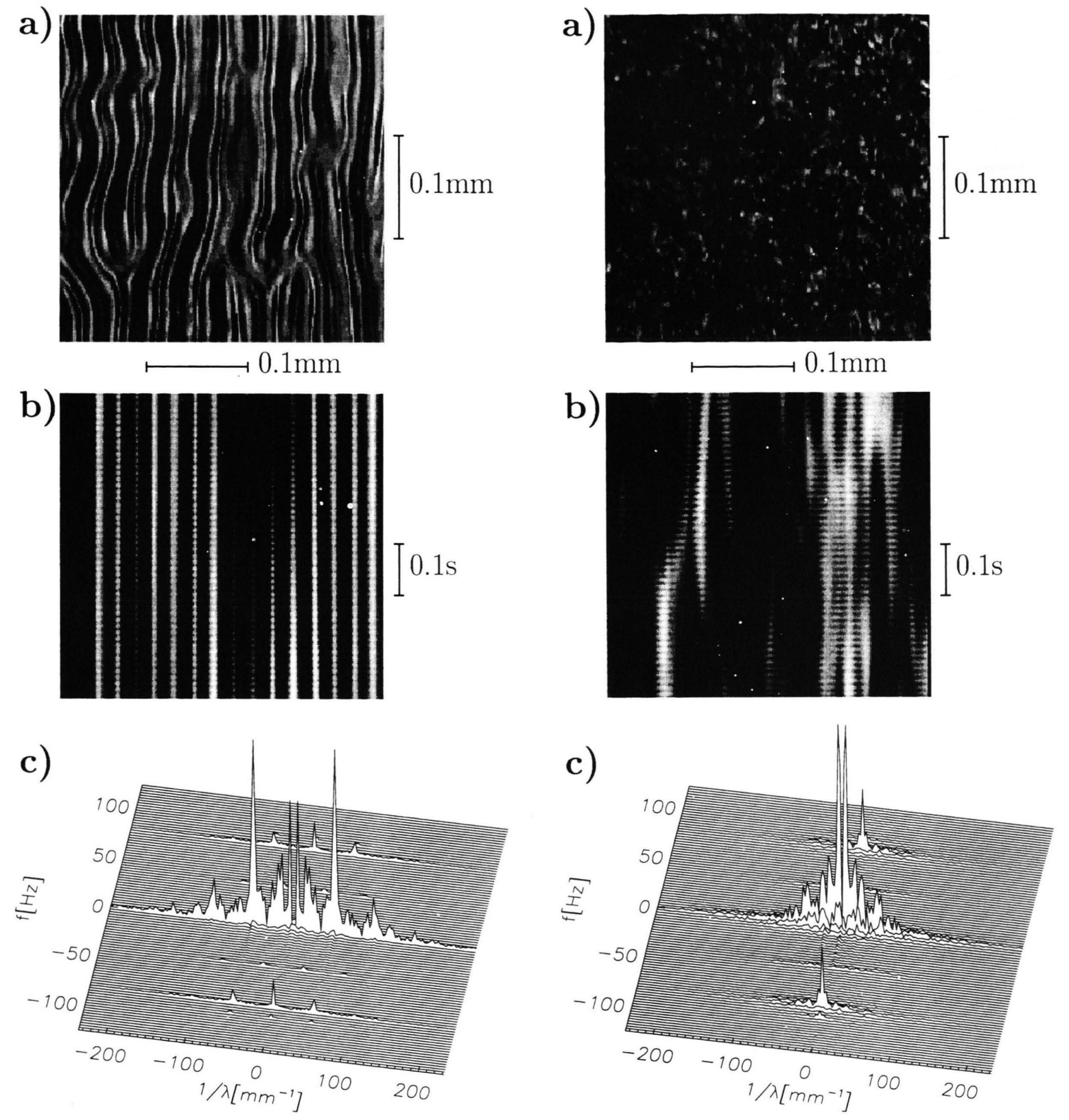

Fig. 3. Fluctuating conductive rolls, $T=30^{\circ} \mathrm{C}, f=40 \mathrm{~Hz}$ $\mathrm{SQ}, U=33.0 \mathrm{~V}, \mathrm{a}-\mathrm{c}$ ) as in Figure 2.

Fig. 4. Dynamic scattering mode, $T=30^{\circ} \mathrm{C}, f=40 \mathrm{~Hz} \mathrm{SQ}$, $U=65.0 \mathrm{~V}, \mathrm{a}-\mathrm{c}$ ) as in Figure 2.

the driving voltage, reflecting periodic oscillations of the director field with twice the excitation frequency overlayed over the stationary deformation mode. In addition to these short-time oscillations, the structure shows pronounced long-term dynamics, i. e. fluctuations of the roll texture in time.

Figure 4 shows images in the DSM region. Here, we cannot extract characteristic wavelengths, the stationary spectrum is rather broad. In addition, sharp maxima appear at $\left(k=0, f= \pm 2 f_{0}\right)$, i. e. the integral transmitted intensity oscillates with $2 f_{0}$. This cannot be explained in terms of optical refraction effects, as 
a)

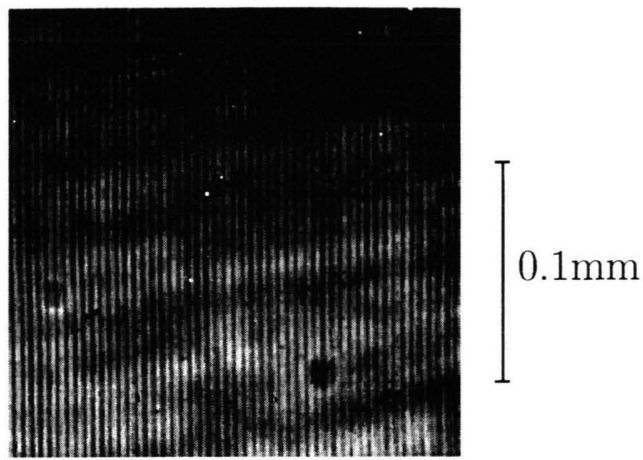

b)

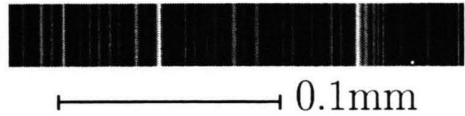

c)
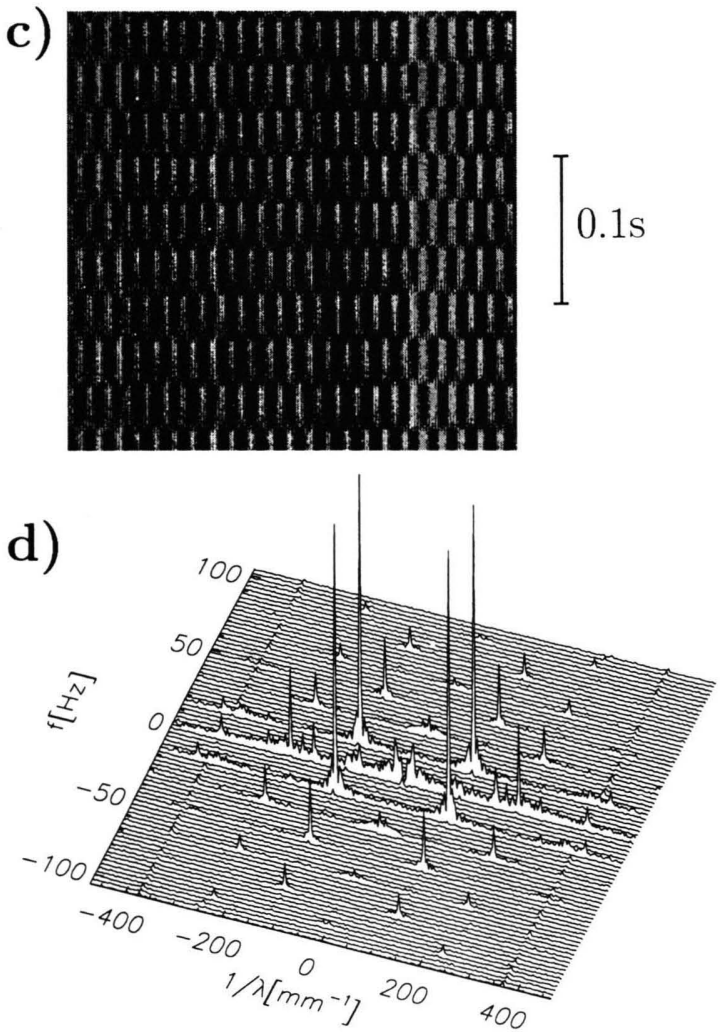

Fig. 5. Normal dielectric rolls, $T=15.8{ }^{\circ} \mathrm{C}, f=16 \mathrm{~Hz}$ $\mathrm{SQ}, U=56.3 \mathrm{~V}$, a) stationary optical image $(x, y)$, b) time average of the $(x, t)$ image in Fig. 5c, c) spatio-temporal image $(x, t)$, cut along the director easy axis, d) 2D Fourier transform of the $(x, t)$ image.

pure refraction leaves the total transmitted intensity unaffected. Rather it indicates that locally the director twists out of the tilt plane (given by rubbing direction and cell normal). In that case, normally incident light polarized along $x$ is no longer an optical eigenmode in the nematic layer. Due to the additional high tilt deformation the wave guiding condition breaks down, the transmitted light becomes elliptically polarized, which leads to interference effects at the analyzer. The transmitted intensity is then modulated in time by the director oscillations. One can show that by removing the analyser. In presence of only one polarizer, the temporal intensity modulations disappear.

The most pronounced effects of time resolved pictures are found in the dielectric regime, above $f_{c}$. As a compromise between camera sensitivity (exposure time) and satisfactory time resolution during the excitation periods, these measurements were performed at $15.8^{\circ} \mathrm{C}$, where the cut-off frequency was $15 \mathrm{~Hz}$. The stationary picture at onset of pattern formation is a low contrast periodically modulated stripe texture (DR). A typical transmission image of DR is seen in Figure 5a. The microscopic focus plane again was chosen at the upper boundary of the nematic layer. The pattern appears most intensive in the vicinity of that plane but is not very sensitive to variations of the focus.

In the time-resolved cross sections (Fig. 5c), the contrast is much increased with respect to the stationary pattern, the wavelength is doubled. In each halfperiod of the driving field, a pattern with wavelength $\lambda_{0}=1 / k_{0}$ is formed. In subsequent half-periods, the pattern is shifted spatially by $1 / 2 \lambda_{0}$, forming a "chessboard" like image in the $(x, t)$ presentation. The corresponding FT in Fig. 5d has four dominant peaks at positions $\left( \pm k_{0}, \pm f_{0}\right)$. The choice of a different height of the microscopic focus does not change this picture qualitatively.

The relative displacement of the momentary texture in subsequent half-cycles of the AC field is such that the positions of intensity maxima alternate with those of intensity minima. The optical contrast, averaged over a time period $T_{0}=1 / f_{0}$, leaves only a small contrast modulation with half the periodicity of the instant images. This is reflected in the FT spectrum by the small peaks at $\pm 2 k_{0}$ on the $f=0$ axis. A numerical time average of the $(x, t)$ image of Fig. $5 \mathrm{c}$ is depicted in Figure 5b. The wavelength in this time averaged line scan camera output coincides with that of the stationary pattern (5a) as expected.

A similar relation between time resolved and averaged optical image as for DR is also observed in the chevron textures where the short wavelength observed in the time averaged image corresponds to $\lambda_{0} / 2$. The long-wavelength secondary structure is 
a)
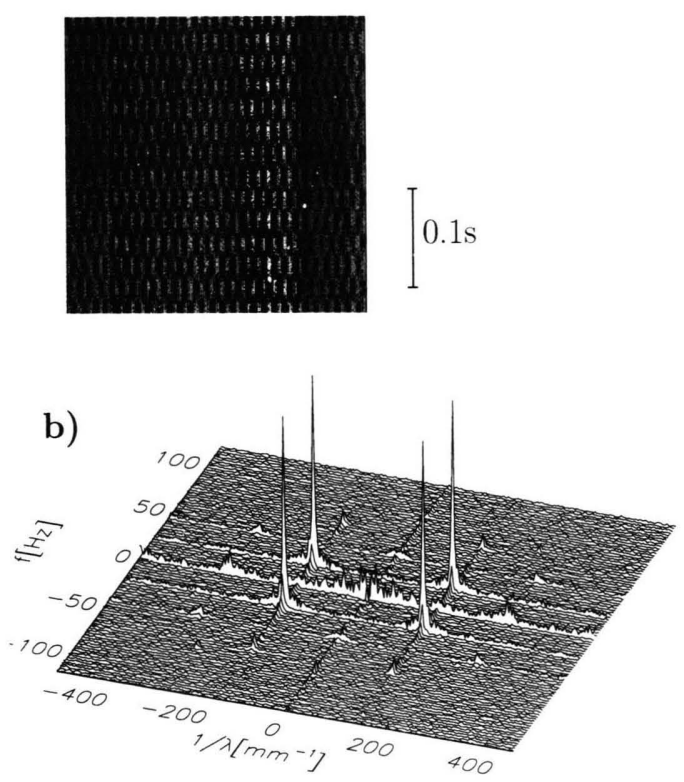

Fig. 6. Normal dielectric rolls, $T=16.6{ }^{\circ} \mathrm{C}, f=22 \mathrm{~Hz}$ sine, $U=63 \mathrm{~V}$, a) spatio-temporal image $(x, t)$, cut along the director easy axis, b) 2D Fourier transform of the $(x, t)$ image.

connected with an additional periodic modulation of the wave vector of the short rolls.

The effects are qualitatively similar for sine and square wave excitation. Figure $6 a$ shows dielectric rolls driven by a sine electric field, and Fig. $6 \mathrm{~b}$ the corresponding Fourier spectrum. Again, the dominating peaks are found at the driving frequency $\left(f_{0}=22 \mathrm{~Hz}\right)$ and wave number $k_{0}$ corresponding to the periodicity of the director field. With sine excitation, the optical response is almost sinusoidal in time, higher harmonics in the time domain are very small, only the $3 f_{0}$ peak is above experimental noise level. At the $f=0$ axis, again the $k_{0}$ peaks are missing. The stationary pattern has the wave number $2 k_{0}$.

DR textures, as observed in conventional microscopic experiments (often performed in the $\mathrm{kHz}$ range), represent such time averages of high contrast instant images. The spatial director modulation $\lambda_{0}$ corresponds to the periodicity of the intensity modulation in the momentary images. Its wavelength is twice that of the averaged textures. More information on the director dynamics is found from the symmetry of the Fourier transforms. All Fourier peaks are found at positions $\left(n k_{0}, m f_{0}\right)$ with $n+m$ even. This is a consequence of the symmetry in the spatio-temporal image in Figs. $5 \mathrm{c}$ and $6 \mathrm{a}$ where $I(x, t)=I\left(x+\lambda_{0} / 2, t \pm T_{0} / 2\right)$. The optical pattern is only shifted by $\lambda_{0} / 2$ along $\mathrm{x}$ in subsequent halfcycles. Therefore the phase insensitive optical contrast of the pattern is periodic with $T_{0} / 2$. Our experimental results are in full accordance with classical theory [7] yet qualitatively different from those reported by Schneider et al. [9] who measured an asymmetric response of the optical contrast of DR with respect to the driving AC field in their samples; their optical contrast curves have a period $T_{0}$. They explained such an asymmetry with possible influences of flexoelectric terms. From theoretical considerations, flexoelectric terms should not produce an asymmetry of optical contrast in our cells (antiparallely rubbed, symmetric anchoring conditions of the director at both glass plates). We consider two momentary pictures of the director field, at times $t_{0}$ and $t_{0}+T_{0} / 2$. At time $t_{0}+T_{0} / 2$, the electric field has changed its sign, $E\left(t_{0}+T_{0} / 2\right)=-E\left(t_{0}\right)$, but when the cell is rotated by $180^{\circ}$ about the $y$ axis, the physical situation is equivalent to that at time $t_{0}$ (even in pretilt cells), when the director deflection $\theta(x, z)$ is replaced by $-\theta(x, z)$. However, the sign of $\theta$ is irrelevant for the optical contrast in the transmission texture. A flexoelectric term might, however, change the spatial periodicity of the images, but such an effect has not been observed in our samples.

Before we perform simulations of the optical characteristics of DR, we discuss now the time dependence of images. Instead of the optical contrast, we will analyze the $\lambda_{0}$ peak intensity. Higher harmonics of the $\lambda_{0}$ peaks in Figs. 5d and $6 \mathrm{~b}$ are rather small, the spatial modulation of the optical image is rather sinusoidal both for square and sine wave excitation. For square wave excitation, the $k_{0}$ Fourier peak of the optical images has higher harmonics in the time domain, i. e. the temporal modulation is not sinusoidal. Figure 7 depicts the time dependence of the $\lambda_{0}$ peak intensity $A_{1}$ obtained from a time series of $1 D$ spatial Fourier transforms. We show the absolute magnitude which faciliates a comparison with data in [9], actually the sign alters between subsequent half-cycles. Within experimental error, the period of the absolute amplitudes is $T_{0} / 2$ in both cases[14] (while that of the pattern is $T_{0}$ ). The dashed curves in Fig. 7 represent a 


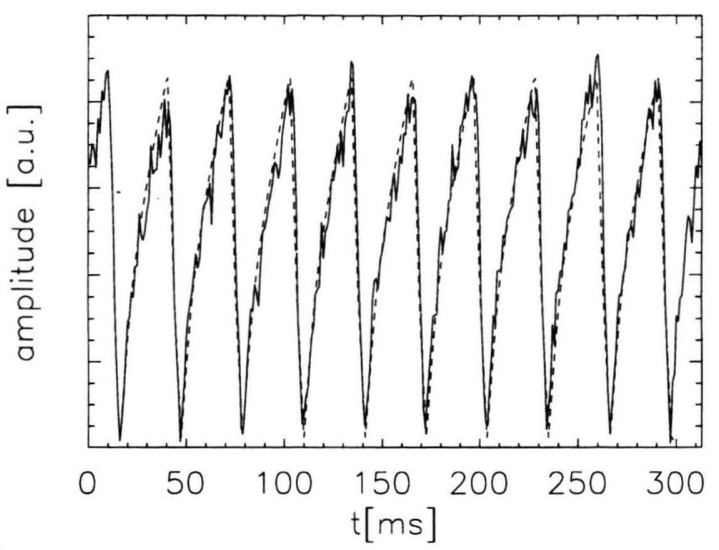

a)

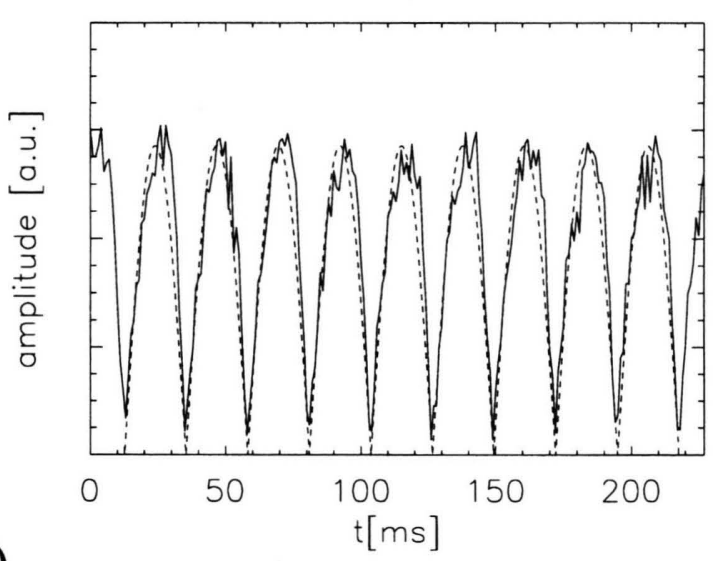

b)

Fig. 7. Time dependent intensity modulation amplitudes of the ground mode $\lambda_{0}$ for square wave (a) and sine wave (b) excitation, extracted from the images in Figs. 5 and 6, resp. The dashed curves correspond to (1) with the parameters given in the text (a) and a pure sine function (b).

time dependence in terms of exponentials (Eq. (1), see below) for rectangular excitation and a sine function for sine excitation. If the modulation amplitude were linearly related to the director deflection amplitude, one could directly determine the director dynamics from these curves. However, this is only a rough approximation, as the optical simulations below will show.

\section{Numerical Simulations}

One can simulate the relation between the observed optical pattern and the director field for the dielectric rolls. The relation between director and intensity modulations is not straightforward. Incident parallel light is deflected in the optically anisotropic and inhomogeneous medium. Several attempts to calculate light propagation in modulated nematic layers have been reported [15]- [22]. These approaches are more or less approximative and use slightly different concepts.

We refer to the algorithm proposed by Rasenat [20] who computed numerically the propagation of individual light rays in a periodically deformed nematic layer using Fermat's principle. The deflection angle of light rays in the dielectric rolls turns out to be very small. Minor differences might be found if an alternative approach is used, but as the purpose of the following discussion is only a qualitative demonstration, these differences are not essential in the discussion of the intensity profiles.

We assume a model director field

$$
\begin{aligned}
& \boldsymbol{n}(x, z)=(\cos \theta(x, z), 0, \sin \theta(x, z)) \\
& \text { with } \theta=\theta_{0} \cos \left(2 \pi x / \lambda_{0}\right) \sin (\pi z / d) .
\end{aligned}
$$

This corresponds to the ground mode (in $z$ ) of an inplane modulated splay-bend deformation, which is a reasonable approximation near the threshold field. Figure $8 \mathrm{a}$ visualizes the propagation of probe light rays. The two horizontal bars give the upper and lower boundaries of the nematic layer embedded in a glass cell. The director modulation is sketched by the sine graph. Refractive indices $n_{e}=1.65278, n_{o}=1.5000$ for Mischung 5 at $20^{\circ} \mathrm{C}$ and $n=1.54$ for the glass plates have been assumed. In the vicinity of the upper boundary of the nematic layer, the light rays are bundled near the positions $x=(n+1 / 4) \lambda_{0},(n=1,2,3 \ldots)$. The intensity shown in the upper graph was calculated numerically. It represents the distribution density of $10^{4}$ rays taken at the upper edge of the nematic layer. Note that the presentation in Fig. 8 is stretched considerably in the horizontal, the nematic layer thickness is much larger than the lateral periodicity of the director field.

Figure 9 depicts the Fourier coefficients $A_{n}(n=$ $\{1 . .4\})$ in the expansion $\sum A_{n} \sin 2 \pi n x / \lambda_{0}$ of the optical intensity profiles as a function of the director deflection angle $\theta_{0}$. The ground mode $(n=1)$ is dominant, in particular at low deflection angles. However, the simulation shows that the relation between its intensity and the deflection amplitude is nonlinear even at small $\theta_{0}$. 

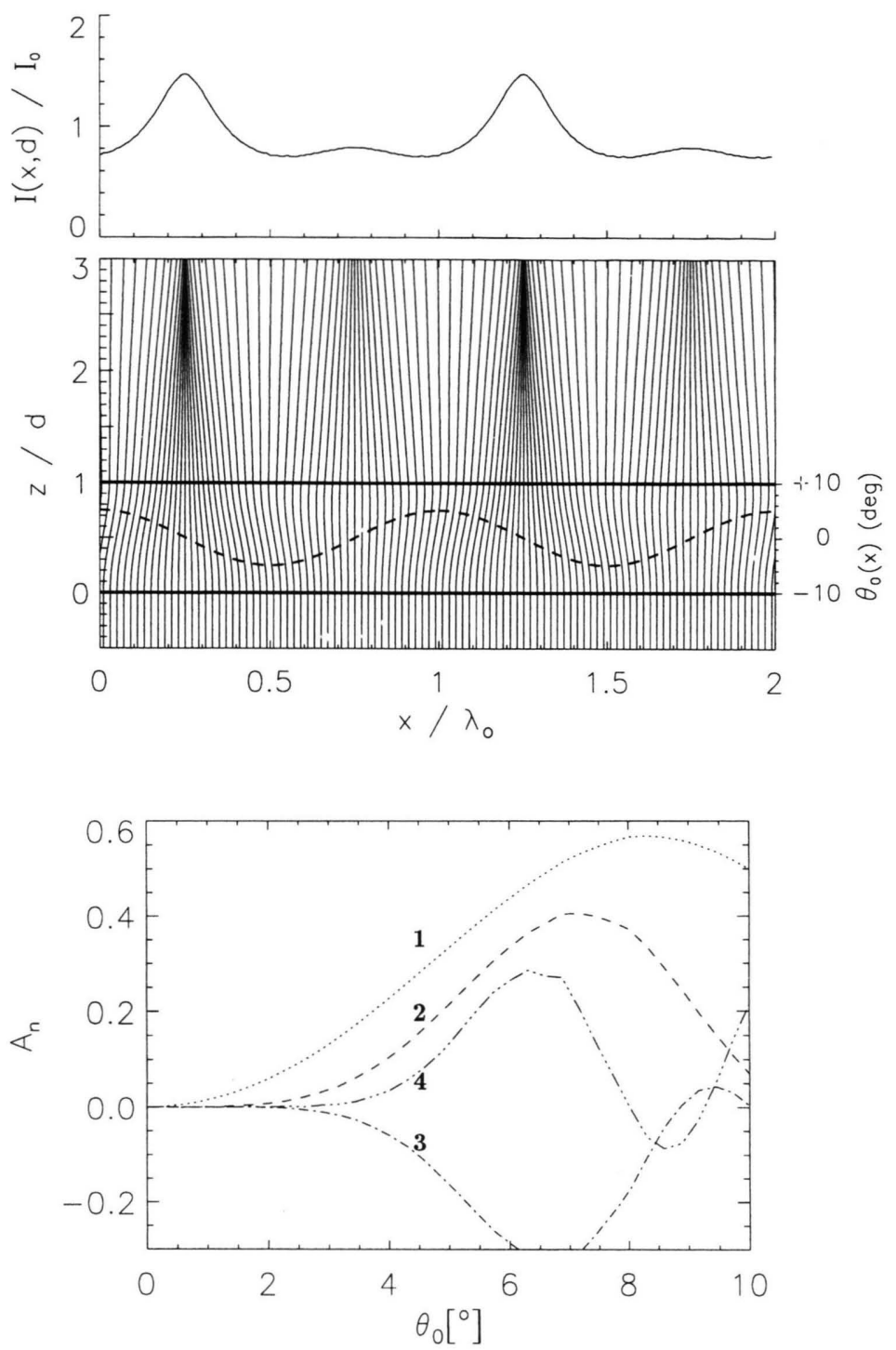

Fig. 8. Propagation of normally incident light rays in a nematic layer with sinusoidally modulated tilt along $x$, tilt amplitude $\theta_{0}=5^{\circ}$, $d=50 \mu \mathrm{m}, \lambda_{0}=10 \mu \mathrm{m}$ from the application of Fermat's principle. At the top we show the transmission intensity calculated from the density of $10^{4}$ light rays. The profile is taken at the upper edge of the nematic layer.
Fig. 9. Growth of the first four Fourier coefficients of the intensity profiles for different $\theta_{0}$.
Figure 8 corresponds to a momentary picture of the director field. With AC excitation, we have to allow for the director field dynamics. When the director modulation changes its sign (after one half-period of the excitation field), this corresponds to a shift of the intensity profile by $\lambda_{0} / 2$ along $x$, i.e. the new maxima appear in the middle between those of the previous half-period. For a calculation of the optical $x, t$ image we have chosen model time dependences of $\theta_{0}$. A sinusoidal response $\theta_{0}(t)=\theta_{\max } \sin \left(2 \pi f_{0} t\right)$ was assumed to describe the director dynamics at sine AC excitation. Figure 10 shows the Fourier spectrum calculated from the time dependent intensity profiles.
This spectrum agrees well with the experimental spectrum for sine excitation (Figure 6b). For square wave excitation, we have assumed the function

$$
\begin{gathered}
\theta_{0}(t) \propto\left[\left(1+e^{-a_{1}}\right) e^{2 a_{2} t / T_{0}}-\left(e^{-a_{1}}+e^{a_{2}-a_{1}}\right) e^{2 a_{1} t / T_{0}}\right] \\
0<t<T_{0} / 2
\end{gathered}
$$

with $\theta_{0}\left(t+T_{0} / 2\right)=-\theta_{0}(t)$. Such a time dependence was derived by Smith et al. [7]. The parameters $a_{1}$ and $a_{2}$ depend on the frequency and strength of the driving field and wavevector $\lambda_{0}$. A simulated optical $x, t$ image $\left(2 \lambda_{0} \times 2 T_{0}\right.$ clip $)$ is depicted in Fig. 10 (bottom). In 

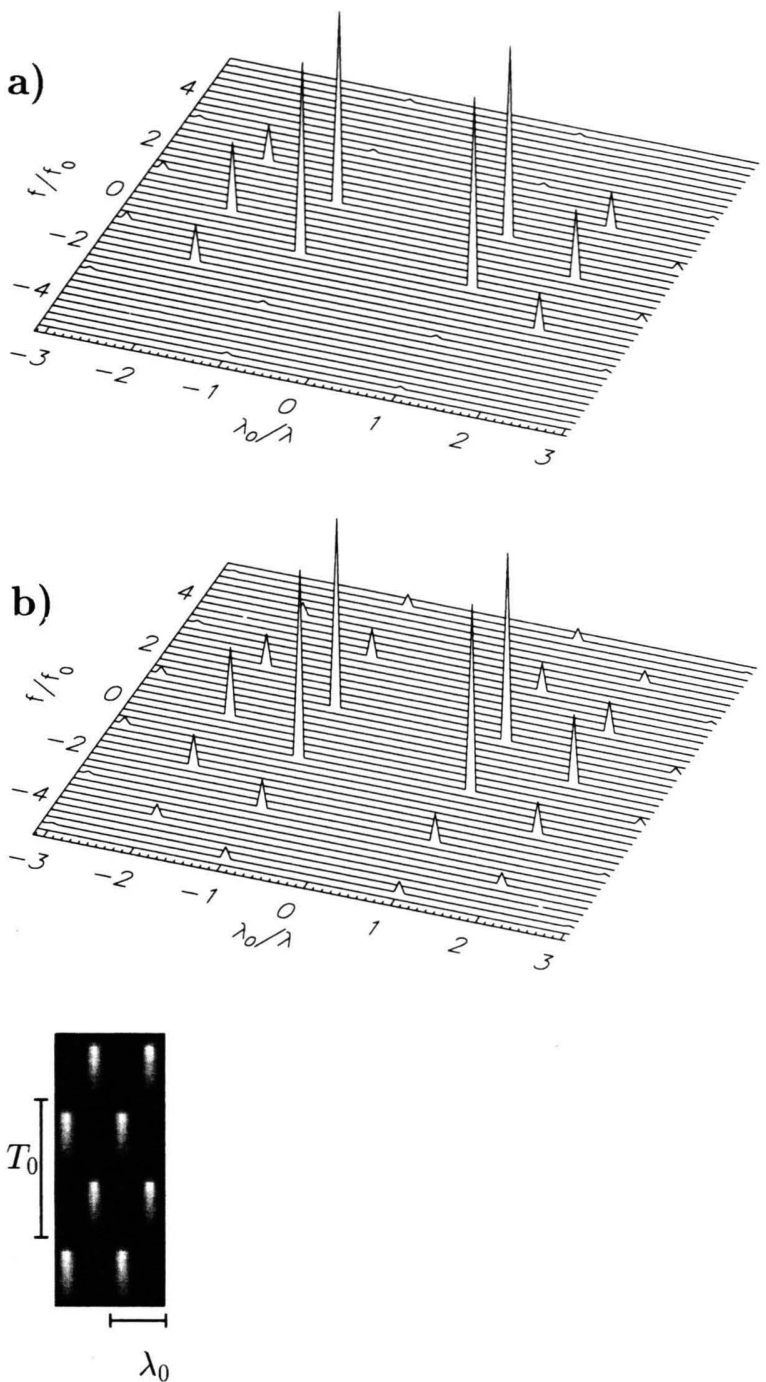

Fig. 10. Fourier transforms of simulated images for sine (a) and square wave (b) excitation. Presentation as in Figs. 5d, 6b. The chessboard image visualizes the spatio-temporal pattern simulated for square wave excitation (see text).

the simulation we have used a parameter set $a_{1}=0.8$ and $a_{2}=-3.8$ and a modulation amplitude of $5^{\circ}$. Fig. 10b shows the Fourier spectrum corresponding to this image. It agrees well with the experimental image $5 \mathrm{~d}$. For both excitations, time averaging leads to a doubled wavevector for the 'stationary' image, seen from the $\pm 2 k_{0}$ peaks on the $f=0$ axis in images $10 a, b$.

\section{Summary}

We have recorded optical images of electroconvection patterns of nematics in a planar cell, time resolved during the excitation field cycles of a driving AC voltage. The symmetry of the temporal modulations has been revealed. In the investigated cells, no asymmetries between the two half-cycles of the driving field were observed in the conduction regime. The time resolved images confirm predictions of the standard theory on the dynamical behaviour of the director field in EHC.

In the DSM patterns, the director field is no longer planar but escapes the tilt plane. The out-of-plane twist is time modulated with the driving field frequency.

A time resolved analysis of the convection rolls in the dielectric regime in combination with a numerical simulation of light propagation in the anisotropic, periodically deformed nematic medium was performed to elucidate the nature of the optical textures and their relations to the director modulation. Differences in the director response to square and sine wave excitation have been shown. We have demonstrated that the low contrast dielectric roll patterns observed in conventional microscopy arise from averaging of high contrast instant images. The momentary images reflect the wavelength $\lambda_{0}$ of the spatial director modulation, while the time averaged images are characterized by $\lambda_{0} / 2$.

In view of the fact that the experimental evidence of the anisotropic standard mechanism [7] in the dielectric regime has been questioned recently [23], our results confirm this mechanism in full agreement with the standard theory [7]. In particular, the optical contrast modulation in time (as defined in [9]) is found to be periodic with $T_{0} / 2$, i.e. no difference was found between the half-cycles of the driving field. An introduction of flexoelectric terms is not necessary for the explanation of our experimental data. 
This study was supported by the DFG with grant STA 425/3-1. The authors acknowledge fruitful comments to this manuscript by I. Rehberg.

[1] R. Williams, J. Chem. Phys. 39, 384 (1963). A. P. Kapustin and L. K. Vistin, Kristallografija 10, 118 (1965).

[2] L. Kramer and W. Pesch, Annu. Rev. Fluid Mech. 17, 515 (1995). L. Kramer and W. Pesch, Electrohydrodynamic Instabilities in Nematic Liquid Crystals in "Pattern formation in Liq. Cryst." ed. A. Buka and L. Kramer, Springer-Verlag, New York 1995, and refs. therein.

[3] E. F. Carr, J. Chem. Phys. 38, 1536 (1963), 39, 1979 (1963), 42, 738 (1969); Mol. Cryst. Liq. Cryst. 7, 253 (1969). W. Helfrich, J. Chem. Phys. 51, 4092 (1969), Mol. Cryst. Liq. Cryst. 21, 187 (1973).

[4] E. Dubois-Violette, P. G. de Gennes, and O. Parodi, J. de Physique 32, 305 (1971).

[5] W. Zimmermann and L. Kramer, Phys. Rev. Lett. 55, 402 (1985). E. Bodenschatz, W. Zimmermann, and L. Kramer, J. Phys. Paris 49, 1875 (1988).

[6] M. Treiber and L. Kramer, Mol. Cryst. Liq. Cryst. 261, 303 (1995).

[7] I. W. Smith, Y. Galerne, S. T. Lagerwall, E. DuboisViolette, and G. Durand, J. Physique Coll. C1, 237 (1975).

[8] S. Kai, S. Wakabyashi, and M. Imasaki, Phys. Rev. A 33, 2612 (1986).

[9] U. Schneider, M. de la Torre Juarez, W. Zimmermann, and I. Rehberg, Phys. Rev. A 46, 1009 (1992).

[10] I. Rehberg, S. Rasenat, J. Fineberg, M. de la Torre Juarez, and V. Steinberg, Phys. Rev. Lett. 61, 2449 (1988).

[11] U. Schuhmacher, Dissertation Halle 1987.
[12] H. Amm, Diplomarbeit Leipzig 1997.

[13] H. Amm, U. Behn, Th. John, and R. Stannarius, Proc. $16^{\text {th }}$ Int. LC Conference Kent 1996, Mol. Cryst. Liq. Cryst. 304, 525 (1997).

[14] Very small (non-systematic) alternations in the amplitudes as indicated in Fig. 7a may appear as a consequence of a slightly inhomogeneous background. In that case, the amplitude of the FT coefficient may be sensitive to the shift of the pattern by $\lambda_{0} / 2$.

[15] L. K. Vistin, A. Yu. Kabaenkov, and S. S. Yakovenko, Sov. Phys. - Crystallogr. 26, 70 (1981).

[16] K. Kondo, A. Fukuda, and E. Kuze, Japan. J. Appl. Phys. 20, 1779(1981). K. Kondo, A. Fukuda, E. Kuze, and M. Arakawa, Japan. J. Appl. Phys. 22, 394 (1983).

[17] H. Richter, S. Rasenat, and I. Rehberg, Mol. Cryst. Liq. Cryst. 222, 219 (1992).

[18] I. Rehberg, F. Horner, and G. Hartung, J. Stat. Phys. 64, 1017 (1991).

[19] V. S. Mylnikov, Opt. Spectrosc. 56, 177 (1984).

[20] S. Rasenat, Dissertation, Bayreuth 1990. S. Rasenat, G. Hartung, B. L. Winkler, and I. Rehberg, Exp. Fluids 7, 412 (1989).

[21] J. A. Kosmopoulos and H. M. Zenginoglou, Appl. Optics 26, 1714 (1987).

[22] A. Joets and R. Ribotta, Opt. Commun. 107, 200 (1994); J. Physique I 4, 1013 (1994).

[23] L. M. Blinov and V. G. Chigrinov, Electrooptic effects in liquid crystal materials, Springer-Verlag, New York 1994. 